\title{
Envelhecimento Funcional e Capacidade para o Trabalho entre Trabalhadores Atendidos pela Atenção Básica
}

\section{ENVEJECIMIENTO FUNCIONAL Y CAPACIDAD DE TRABAJO ENTRE LOS TRABAJADORES ANTEDIDOS EN LA ATENCIÓN PRIMARIA}

Rachel Beatriz Cardoso Valério', Fabiana Caetano Martins Silva e Dutra²

1. Terapeuta Ocupacional pela Universidade Federal do Triângulo Mineiro. Estudante do Núcleo de Estudos e Pesquisas em Trabalho, Participação Social e Saúde (NETRAS), Uberaba, MG, Brasil.

2. Professora Adjunta do Departamento de Terapia Ocupacional e do Programa de Pós-Graduação em Atenção à Saúde. Universidade Federal do Triângulo Mineiro. Pesquisadora do Núcleo de Estudos e Pesquisas em Trabalho, Participação Social e Saúde (NETRAS), Uberaba, MG, Brasil.

\begin{abstract}
RESUMO
0 envelhecimento da população trabalhadora estimula estudos sobre funcionalidade, saúde e capacidade para o trabalho dos trabalhadores. Objetivos: descrever, comparar e correlacionar capacidade para o trabalho e capacidade funcional em trabalhadores jovens e trabalhadores mais velhos. Metodologia: estudo transversal que avaliou capacidade funcional e capacidade para o trabalho (CT) de 94 trabalhadores divididos em dois grupos: grupo 1 formado por trabalhadores com até 44 anos; grupo 2 com trabalhadores com 45 anos ou mais. Resultados: maioria eram mulheres, com ensino médio incompleto. Grupo 1 tinha média de 28,57 anos e maioria era solteira. Grupo 2, a maioria era casada e tinha média de 51,34 anos. Trabalhadores apresentaram boa CT e leve incapacidade. Não houve diferença para incapacidade $(p=0,372)$ e CT $(p=0,084)$ entre os grupos. Houve correlação significativa entre $\mathrm{CT}(\mathrm{p}=0,003)$ e incapacidade $(\mathrm{p}=0,000)$, sendo correlação moderada no grupo $1(\mathrm{r}=-0,419)$ e forte no grupo $2(\mathrm{r}=-0,706)$. Conclusão: os resultados apontam que trabalhadores mais velhos mantém sua capacidade para o trabalho, apesar do processo de envelhecimento. Também se confirmou a correlação entre capacidade para o trabalho e capacidade funcional.
\end{abstract}

Palavras chave: AVALIAÇÃO DA CAPACIDADE DE TRABALHO, SAÚDE DO TRABALHADOR, ENVELHECIMENTO DA POPULAÇÃO, ATIVIDADES COTIDIANAS.

\section{RESUMEN}

El envejecimiento de la población trabajadora estimula los estudios sobre funcionalidad, salud y capacidad de trabajo de los trabajadores. Objetivos: describir, comparar y correlacionar capacidad de trabajo y la capacidad funcional de los trabajadores jóvenes y los trabajadores mayores. Metodología: Estudio transversal de la evaluación de la capacidad funcional y capacidad de trabajo (CT) de 94 trabajadores divididos en dos grupos: grupo 1 formado por trabajadores de hasta 44 años; el grupo 2 con los trabajadores de 45 o más años de edad. Resultados: la mayoría eran mujeres con educación secundaria incompleta. El grupo 1 tenía un promedio de 28,57 años y la mayoría eran solteros. Grupo 2, la mayoría estaban casados y tenían un promedio de 51.34 años. Los trabajadores mostraron buena TC y leve incapacidad. No hubo diferencias para la incapacidad $(p=0,372)$ y CT $(p=0,084)$ entre los grupos. Hubo una correlación significativa entre la TC $(\mathrm{p}=0,003)$ y discapacidad $(\mathrm{p}=$ $0,000)$ y la correlación moderada en el grupo $1(\mathrm{r}=-0,419)$ y fuerte en el grupo $2(r=-0,706)$. Conclusión: Los resultados indican que los trabajadores mayores mantienen su capacidad de trabajo, a pesar del proceso de envejecimiento. También confirmó la correlación entre la capacidad de trabajo y la capacidad funcional.

(Cardoso R, Martins F, 2016. Envejecimiento Funcional y Capacidad de Trabajo entre los Trabajadores Atendidos en la Atención Primaria. Cienc Trab. Sep-Dic; 18 [57]: 190-195).

Palabras clave: EVALUACIÓN DE LA CAPACIDAD DE TRABAJO, SALUD DEL TRABAJADOR, ENVEJECIMIENTO DE LA POBLACIÓN, ACTIVIDADES COTIDIANAS.

\section{INTRODUÇÃO}

O Brasil apresenta um dos mais agudos processos de envelhecimento populacional entre os países mais populosos. A proporção

\section{Correspondencia / Correspondence:}

Fabiana Caetano Martins Silva e Dutra

Departamento de Terapia Ocupacional, Instituto de Ciências da Saúde, Universidade Federal do Triângulo Mineiro

Av. Getúlio Guaritá, 159, sala 329, 3º piso, Centro Educacional. Uberaba, MG, CEP 38025-440, Brasil

Tel.: +553430761258

e-mail: fabiana@to.uftm.edu.br

Recibido: 10 de Octubre de 2016 / Aceptado: 22 de Noviembre de 2016 de pessoas idosas com 60 anos ou mais, aumentou de 15,5 milhões em 2001, para 23,5 milhões em 2011. ${ }^{1} 0$ envelhecimento é um processo da vida que se caracteriza pelo conjunto de modificações, morfológicas, fisiológicas, bioquímicas, psicológicas e pela perda progressiva da capacidade de adaptação ao meio ambiente afetando a capacidade do indivíduo em realizar tarefas cotidianas, como atividades de vida diária e atividades de trabalho. ${ }^{2}$ Este cenário tem impacto nos recursos econômicos para pagamentos de pensões e aposentadorias, mas principalmente nos gastos em saúde, que representarão um dos maiores desafios fiscais para o país. $^{3,4}$

A Organização Mundial da Saúde (OMS) tem se preocupado com o trabalhador em fase de envelhecimento. Discussões relacionadas ao envelhecimento funcional constituem prioridade no campo da saúde do trabalhador e faz com que a capacidade para o trabalho seja um importante indicador por abranger aspectos relativos à 
saúde física, bem-estar psicossocial, competência individual e condições de trabalho. ${ }^{4}$ A capacidade para o trabalho é definida como a capacidade que o profissional tem pra realizar suas atividades laborais de acordo com seu estado de saúde físico e mental e com as exigências do próprio trabalho. ${ }^{5}$ Estima-se que o declínio da capacidade para o trabalho ocorra após os 45 anos de idade e se acentue gradativamente após os 50 anos, além de receber influências de doenças e acidentes, dos aspectos sócio demográficos, das condições de trabalho, do estilo de vida do indivíduo. ${ }^{5-9}$

Estudos tem indicado que a perda da capacidade funcional, com consequente aumento do número das aposentadorias por invalidez $\mathrm{e}$ aumento do número de afastamentos por tempo prolongado, pode estar associada à baixa capacidade para o trabalho..$^{3,10}$ Neste sentido, a capacidade funcional é de grande importância para a capacidade para o trabalho. Nygard et $\mathrm{al}^{10} \mathrm{em}$ seu estudo sobre a relação da capacidade funcional com a capacidade para o trabalho, se referem à capacidade funcional como sendo a capacidade que o organismo tem para atingir metas estabelecidas, e que a mesma ainda pode ser dividida em níveis físicos, mentais e sociais. A capacidade funcional pode ser considerada a base para a atividade laboral; logo uma capacidade funcional elevada está associada a uma boa capacidade para o trabalho. ${ }^{10}$ Já a redução da capacidade funcional pode comprometer a prática das atividades laborais. ${ }^{6,11}$

Assim, com o envelhecimento da população trabalhadora, temas como aposentadoria, capacidade para o trabalho, saúde e funcionalidade do trabalhador em fase de envelhecimento se tornam importantes objetos de estudos para manter boas condições de saúde, capacidade para o trabalho e funcionalidade do trabalhador. Para Sampaio e Augusto ${ }^{9}$, é necessário aprofundar em mensurações disponíveis, propor novas aferições e intervenções para promover a capacidade para o trabalho, além de potencializar seu uso como desfecho na área da saúde. Diante do exposto, buscou-se avaliar capacidade para o trabalho e capacidade funcional de trabalhadores atendidos pela rede pública de saúde de Uberaba/MG, Brasil, correlacionar capacidade para o trabalho com capacidade funcional, e comparar capacidade para o trabalho e capacidade funcional de trabalhadores adultos com trabalhadores em fase de envelhecimento.

\section{PERCURSO METODOLÓGICO}

Este estudo foi desenvolvido em uma perspectiva observacional, de corte transversal, usando uma abordagem quantitativa, aprovado pelo Comitê de Ética em Pesquisa da Universidade Federal do Triângulo Mineiro (parecer $n^{\circ}$ 2394). Todos os participantes foram informados sobre os objetivos e procedimentos de coleta de dados e aqueles que aceitaram participar voluntariamente do estudo assinaram termo de consentimento livre e esclarecido.

A estimativa de tamanho da amostra considerou um nível de confiança de 95\%; erro máximo desejado de três pontos; e um desvio -padrão médio, estimado em um estudo piloto, igual a 15,5 pontos, totalizando uma amostra mínima de 42 indivíduos por grupo. Foram selecionados por conveniência trabalhadores adultos, atendidos pela rede pública em Unidades Básicas de Saúde, na cidade de Uberaba, MG, Brasil, entre março e outubro de 2014. Os critérios de inclusão eram adultos de ambos os sexos com faixa etária entre 18 e 60 anos. Os participantes deveriam ser trabalhadores ativos no mercado de trabalho, independente da formalidade do trabalho. Foram excluídos aqueles que não apresentaram disponibilidade para participar do estudo, apresentaram condições que impediam ou prejudicavam a compreensão ou fidedignidade das respostas, e que estavam inativos ou afastados das atividades de trabalho. Os participantes foram categorizados em dois grupos: o grupo 1 dos trabalhadores jovens com idade entre 18 e 44 anos; e o grupo 2 composto por trabalhadores em fase de envelhecimento (com mais de 45 anos de idade).

Para caracterização dos participantes, informações sócio demográficas e ocupacionais foram coletadas em um questionário desenvolvido para este estudo composto por questões como sexo, idade, estado civil, escolaridade, ocupação atual e tempo na ocupação.

A capacidade para o trabalho foi mensurada pelo Índice de Capacidade para o Trabalho (ICT) que avalia a capacidade para o trabalho do indivíduo, considerando as demandas físicas e mentais, além dos recursos e condição de saúde segundo a percepção do próprio trabalhador. ${ }^{5} 0$ ICT avalia sete dimensões: capacidade para o trabalho atual comparada com a de toda vida; capacidade para o trabalho em relação às exigências do trabalho; número e doenças diagnosticadas pelo médico; perda estimada para trabalho por doenças; faltas ao trabalho por doenças nos últimos 12 meses; prognóstico próprio da capacidade para o trabalho daqui a dois anos; e recursos mentais. 0 escore varia de 7 a 49 pontos, sendo que de 7 a 27 a capacidade para o trabalho do indivíduo é considerada baixa, de 28 a 36 a capacidade é moderada, de 37 a 43 é boa e de 44 a 49 a capacidade para o trabalho é considerada ótima. ${ }^{5}$

0 Instrumento de Avaliação de Incapacidade II (WHODAS II) foi desenvolvido pela OMS para mensurar incapacidades funcionais relacionada com a saúde em seis diferentes domínios: cognição, mobilidade, autocuidado, atividades diárias, relações interpessoais e participação. ${ }^{12} 0$ WHODAS II examina como as pessoas desenvolvem suas atividades habituais utilizando ou não dispositivo de auxilio, e/ ou ajuda de terceiros. A versão reduzida deste instrumento analisa, em uma escala ordinal de cinco pontos, dificuldades na realização de 12 itens nos últimos 30 dias, onde zero significa nenhuma dificuldade, e cinco que o indivíduo não consegue realizar o item. ${ }^{12} 0$ escore total do WHODAS II varia de zero a 100 pontos, e quanto mais elevada for a pontuação, maior é a incapacidade do indivíduo, levando em consideração a dor, dificuldade, esforço, incômodo e lentidão do indivíduo na realização da tarefa. ${ }^{12}$

Os dados coletados foram submetidos a uma análise descritiva e teste de normalidade. Para a comparação capacidade funcional entre trabalhadores jovens e os trabalhadores em fase de envelhecimento foi utilizado o teste Mann-Whitney e, para comparação da capacidade para o trabalho foi utilizado o teste $t$ de Student. Por fim, empregou-se o Coeficiente de Correlação de Spearman para correlacionar as variáveis capacidade funcional e capacidade para $o$ trabalho entre os trabalhadores jovens e os trabalhadores em fase de envelhecimento. Em todos os testes estatísticos foi ponderado um nível de significância de 5\% e as análises foram realizadas no software SPSS versão 20.0.

\section{RESULTADOS}

A amostra do estudo foi constituída por 94 trabalhadores sendo 49 trabalhadores tinham idade até 44 anos (grupo dos trabalhadores jovens) e 45 trabalhadores tinham idade igual ou maior que 45 anos (grupo dos trabalhadores em fase de envelhecimento). A maioria dos participantes trabalhavam como prestadores de serviço, como domésticas, técnicos em enfermagem, médicos, enfermeiros, agentes de saúde, motoristas, cozinheiros, zeladores, assistentes administrativos e sociais, bibliotecários, carteiros, 
Tabela 1.

Características sócio demográficas dos trabalhadores atendidos pela rede publica de saúde de Uberaba; $n=94$, Uberaba, 2015.

\begin{tabular}{|c|c|c|}
\hline Variáveis & $\begin{array}{c}\text { Trabalhadores } \\
\text { até } 44 \text { anos }(n=49) \\
N(\%)\end{array}$ & $\begin{array}{c}\text { Trabalhadores com } \\
45 \text { anos ou mais }(n=45) \\
N(\%)\end{array}$ \\
\hline \multicolumn{3}{|l|}{ Sexo } \\
\hline Masculino & $18(36,7)$ & $13(28,9)$ \\
\hline Feminino & $31(63,3)$ & $32(71,1)$ \\
\hline \multicolumn{3}{|l|}{ Estado Civil } \\
\hline Solteiro & $30(61,2)$ & $6(13,3)$ \\
\hline Casado & $18(36,7)$ & $28(32,2)$ \\
\hline Separado/Divorciado & $1(2,0)$ & $9(20,0)$ \\
\hline Viúvo & $0(0,0)$ & $2(4,4)$ \\
\hline \multicolumn{3}{|l|}{ Escolaridade } \\
\hline Não Estudou & $1(2,0)$ & $3(6,7)$ \\
\hline Ensino Fundamental Incompleto & $2(4,1)$ & $5(11,1)$ \\
\hline Ensino Fundamental Completo & $2(4,1)$ & $4(8,9)$ \\
\hline Ensino Médio Incompleto & $15(30,6)$ & $13(28,9)$ \\
\hline Ensino Médio Completo & $15(30,6)$ & $9(20,0)$ \\
\hline Ensino Superior Incompleto & $10(20,4)$ & $6(13,3)$ \\
\hline Ensino Superior Completo & $4(8,2)$ & $4(8,9)$ \\
\hline Pós-Graduação & $0(0,0)$ & $1(2,2)$ \\
\hline
\end{tabular}

Tabela 2.

Média da idade, renda familiar, tempo na ocupação atual dos trabalhadores atendidos pela rede publica de saúde de Uberaba; $n=94$, Uberaba, 2015.

\begin{tabular}{|c|c|c|c|c|}
\hline \multirow{2}{*}{ Variáveis } & \multicolumn{2}{|c|}{$\begin{array}{c}\text { Trabalhadores } \\
\text { até } 44 \text { anos }(n=49)\end{array}$} & \multicolumn{2}{|c|}{$\begin{array}{c}\text { Trabalhadores com } \\
45 \text { anos ou mais }(n=45)\end{array}$} \\
\hline & Média & Desvio Padrão & Média & Desvio Padrão \\
\hline Idade (anos) & 28,57 & 7,97 & 51,37 & 4,05 \\
\hline Renda familiar (reais) & 2875,06 & 2296,31 & 3158,22 & 2428,90 \\
\hline $\begin{array}{l}\text { Tempo na ocupação } \\
\text { atual (meses) }\end{array}$ & 31,57 & 32,78 & 132,91 & 133,68 \\
\hline
\end{tabular}

porteiros, vendedores, cuidadores, contadores, entregadores, manicures, mecânicos, nutricionistas, confeiteiros, professores, pedagogos, pintores, policiais, recepcionistas, serviços gerais, soldadores, vidraceiros e servidores públicos.

No grupo dos trabalhadores jovens, a maioria era do sexo feminino $(63,3 \%)$ com média de idade igual a 28,57 anos ( $D P=7,97)$. Em média estes trabalhadores estavam há 34,57 meses na ocupação atual e possuíam renda familiar de $\mathrm{R} \$ 2.875,06$ reais ( $D P=R \$ 2.296,31$ reais). A maioria era solteira $(61,2 \%)$ e relatou ter o ensino médio incompleto ou completo (30,6\% respectivamente).

No grupo dos trabalhadores mais velhos, a maior parte, 71,1\%, era do sexo feminino, com idade variando de 45 a 60 anos ( média de 51,37 anos; $\mathrm{DP}=4,05$ anos). A média da renda familiar desse grupo foi de $\mathrm{R} \$ 2.917,14$ reais $(\mathrm{DP}=2.171,88)$ e estes trabalhadores estavam estáveis na mesma ocupação por, 132,91 meses, o equivalente a 11 anos. A maioria era casada $(62,2 \%)$ e não completou o ensino médio (28,9\%).

A tabela 1 apresenta de forma completa as caracteristicas sócio dos dois grupos de trabalhadores e a tabela 2 apresenta informações detalhadas das variáveis idade, renda e tempo na ocupação.

A média do escore da capacidade funcional do grupo de trabalhadores mais novos e dos trabalhadores em fase de envelhecimento foi 14,85 e 15,75, respectivamente. A análise comparando capacidade funcional entre os dois grupos não identificou diferença estatística significativa $(\mathrm{p}=0,372)$. Este resultado indica que não há diferenças na capacidade funcional destes trabalhadores quando comparados em relação à idade.
Figura 1.

Percentual das categorias do Índice de Capacidade para o Trabalho nos trabalhadores com idade até 44 anos $(n=49)$ e nos trabalhadores com 45 anos ou mais de idade $(n=45)$; Uberaba, 2015.

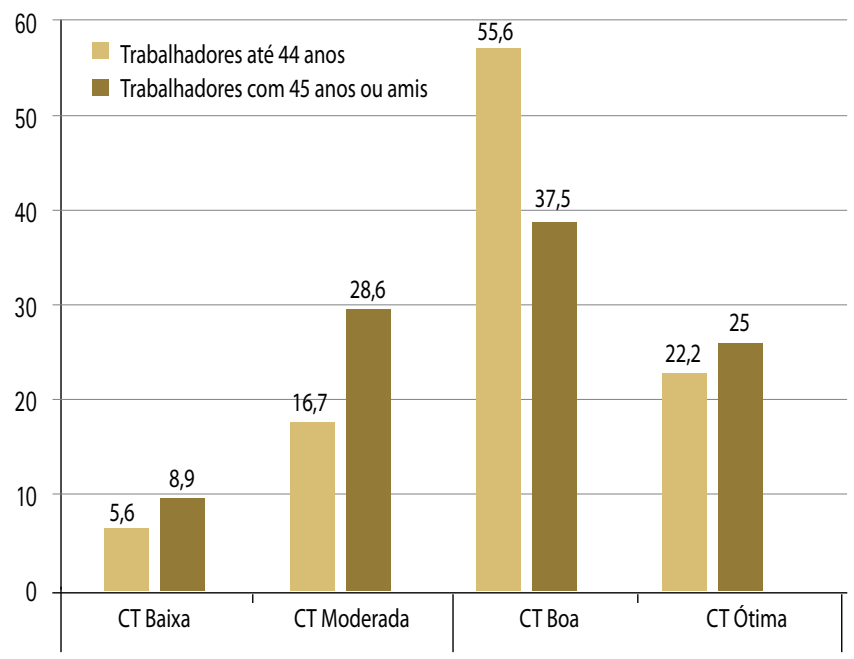

Tabela 3.

Resultado da comparação da capacidade funcional e capacidade para o trabalho entre os grupos de com idade até 44 anos $(n=49)$ e nos trabalhadores com 45 anos ou mais de idade $(n=45)$; Uberaba, 2015.

\begin{tabular}{|c|c|c|c|c|}
\hline & $\begin{array}{c}\text { Trabalhadores até } \\
44 \text { anos }(n=49) \\
\text { Média } \pm D P\end{array}$ & $\begin{array}{l}\text { Trabalhadores com } 45 \\
\text { anos ou mais }(n=45) \\
\text { (Média } \pm D P)\end{array}$ & Valor $p^{1}$ & Significância² \\
\hline $\begin{array}{l}\text { Capacidade } \\
\text { Funcional }^{3}\end{array}$ & $14,86 \pm 3,56$ & $15,76 \pm 4,36$ & 0,372 & NS \\
\hline $\begin{array}{l}\text { Capacidade para } \\
\text { o trabalho }{ }^{4}\end{array}$ & $41,58 \pm 4,45$ & $39,80 \pm 5,47$ & 0,084 & NS \\
\hline
\end{tabular}

${ }^{1} \alpha=0,05 .{ }^{2}$ NS=Não significativo. ${ }^{3}$ Mann-Whitney test. ${ }^{4}$ T-student test.

A análise da capacidade para o trabalho revelou escores médios iguais a 41,58 pontos no grupo de trabalhadores mais novos e 39,80 no grupo de trabalhadores em fase de envelhecimento. De forma categórica (figura 1), 26,5\% dos trabalhadores do grupo 1 tem capacidade para o trabalho considerada ótima, enquanto que no grupo 2, este valor é de $27,1 \%$. Já entre os que possuem capacidade boa, $57,1 \%$ são do grupo de trabalhadores mais novos e 44,4\% entre os trabalhadores em fase de envelhecimento. 16,3\% e 29,8\%, respectivamente, dos participantes têm capacidade para o trabalho considerada moderada. Nenhum trabalhador dos dois grupos foi avaliado com baixa capacidade para o trabalho.

$\mathrm{Na}$ análise comparativa da capacidade para o trabalho entre os dois grupos, não houve diferença estatística significativa ( $p=0,084)$, o que indica que a capacidade para o trabalho também não apresentou diferença quando comparada por idade. A Tabela 3 apresenta a análise comparativa da capacidade funcional e capacidade para 0 trabalho entre os dois grupos de trabalhadores.

Por fim, a Figura 1 apresenta as correlações entre as variáveis capacidade funcional e capacidade para o trabalho. No grupo de trabalhadores jovens, capacidade funcional e capacidade para o trabalho apresentaram uma correlação significativa $(p=0,003)$ e de magnitude moderada $(r=-0,419)$. Esta associação também foi verificada no grupo dos trabalhadores em fase de envelhecimento $(\mathrm{p}=0,000)$, porém com alta magnitude $(\mathrm{r}=-0,706)$, indicando que trabalhadores com maior capacidade funcional são avaliados com melhor capacidade para o trabalho. 
Figura 2.

Correlações entre capacidade para o trabalho e capacidade funcional nos com idade até 44 anos $(n=49)$ e nos trabalhadores com 45 anos ou mais de idade ( $n=45)$; Uberaba, 2015.

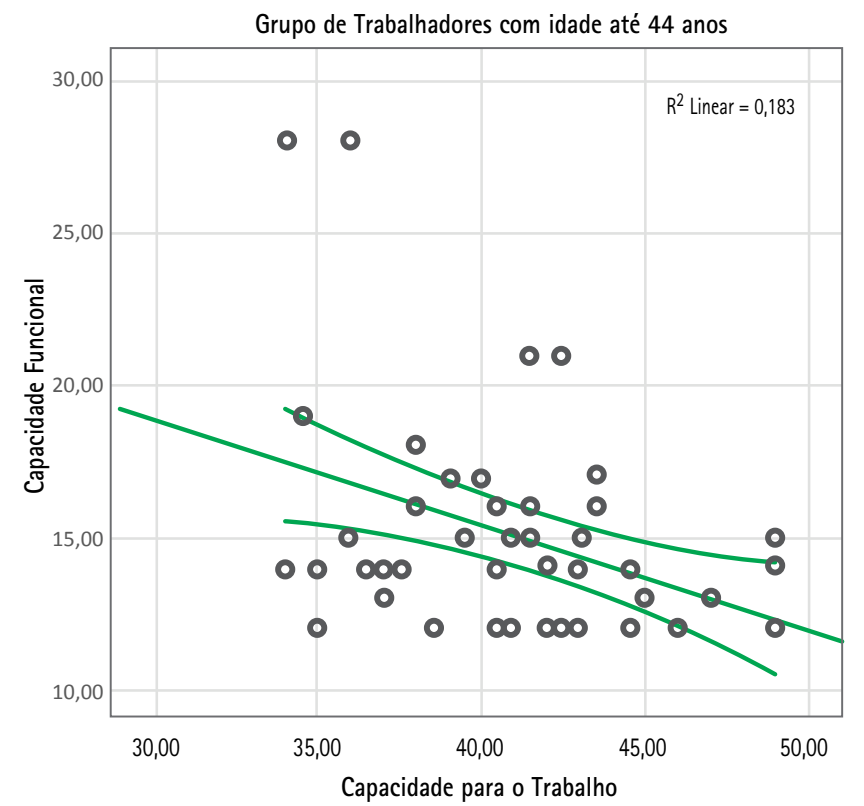

\section{DICUSSÃO}

Os resultados encontrados apontam para uma associação entre capacidade funcional e capacidade para o trabalho confirmando a relação entre saúde, funcionalidade e desempenho em atividades laborais. Ou grupos analisados não apresentaram diferença em relação à capacidade funcional e capacidade para o trabalho indicando que os trabalhadores avaliados mantém sua funcionalidade e capacidade para o trabalho, mesmo nas fases iniciais do processo de envelhecimento. Nos últimos anos, a população brasileira economicamente ativa vem apresentando um profundo processo de envelhecimento e, em poucos anos, a OMS estima que o Brasil ocupe o sexto lugar no ranking dos países com maior número de idosos. ${ }^{1}$ Neste contexto, discutir saúde, funcionalidade e capacidade para o trabalho do trabalhador em fase de envelhecimento se torna importante objeto de estudo na busca de informações para manter boas condições de saúde, capacidade para o trabalho, funcionalidade, aposentadoria e participação social do trabalhador. ${ }^{9}$

Os participantes foram divididos em dois grupos de análise: um grupo formado por trabalhadores com até 44 anos; e um grupo com 45 anos ou mais. Em ambos os grupos foi observado maior percentual de mulheres compondo a amostra. Uma possível explicação para esta amostra essencialmente feminina pode estar associada ao local da coleta de dados que foi realizada em Unidades Básicas de Saúde. No Brasil, o padrão de procura e de uso dos serviços de saúde é determinado por fatores que afetam diferentemente homens e mulheres. Os hábitos de prevenção estão associados mais às mulheres e o uso predominante de serviços de saúde pela população feminina é atribuído a fatores como reprodutividade, pior percepção de saúde e maior incidência de doenças crônicas não fatais entre as mulheres. ${ }^{13}$ Além disso, a taxa de participação feminina na força de trabalho tem aumen-

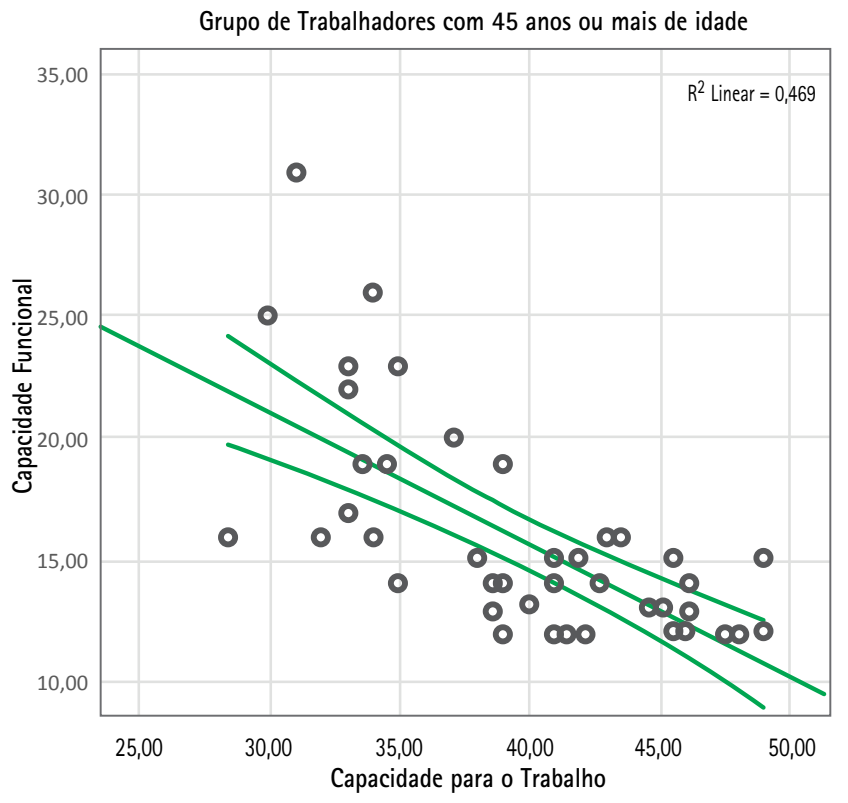

tado bastante no Brasil, independente da formalidade ou não do trabalho exercido. Ainda em 2011, as mulheres representavam $45,4 \%$ do total da população ocupada ${ }^{14}$, o que também pode explicar o grande número de mulheres na população estudada. 0 grupo dos trabalhadores jovens apresentou percentual de escolarização maior do que o grupo dos trabalhadores em fase de envelhecimento, devido à grande expansão educacional que o Brasil tem vivenciado nos últimos anos. Houve um crescimento da cobertura da rede de ensino do país nos últimos 12 anos, o que pôde aumentar acesso ao ensino fundamental e médio, diminuir a evasão escolar e expandir o ingresso de pessoas no ensino superior. Nonato et $\mathrm{al}^{15}$ revelam que os trabalhadores brasileiros que mais se beneficiaram com essa expansão educacional foram os jovens, que tinham entre 18 e 29 anos, o que se assemelha bastante à média de idade dos trabalhadores jovens dessa pesquisa. Atualmente, cerca de 50\% dos trabalhadores brasileiros possuem de 9 a 12 anos de estudo, o que corresponde ao ensino médio incompleto. ${ }^{15}$

No Brasil a utilização do ICT teve inicio na década de 90 e, desde então, esse instrumento é usado em estudos para avaliar a capacidade para o trabalho e seus fatores de risco. Apesar de o ICT ser um instrumento capaz de indicar o quão bem o trabalhador está para realizar sua atividade laboral, constatar e avaliar alterações na capacidade para o trabalho, indicar possiveis intervenções com esse trabalhador e predizer casos precoces de aposentadorias e de absenteísmo, as pesquisas nacionais ainda se concentram em utilizá-lo apenas de forma descritiva. Segundo Tuomi et al5, escores de 7 a 27 pontos indicam baixa capacidade para o trabalho e a necessidade de medidas para sua restauração. Escores de 28 a 36 pontos mostram capacidade para o trabalho moderada e recomenda melhorá-la. Escores de 37 a 43 implicam boa capacidade para o trabalho e medidas para promoção da mesma, e pontuações entre 44 e 49 informam que o individuo está com capacidade para o trabalho ótima, e assim deve mantê-la. 
Neste estudo, em relação à capacidade para o trabalho, não houve diferença significativa entre os trabalhadores dos dois grupos, sendo que em ambos os grupos, a maioria dos participantes foi classificada com boa capacidade para o trabalho. Nos estudos de Moura et $\mathrm{al}^{16}$ sobre a capacidade para o trabalho em trabalhadores da prefeitura de um campus universitário, também observou-se boa capacidade para o trabalho tanto nos funcionários com idade entre 20 e 40 anos, quanto nos trabalhadores com idade acima de 41 anos. Padula et $\mathrm{al}^{17}$ também analisaram a capacidade para $\mathrm{o}$ trabalho de trabalhadores jovens e em fase de envelhecimento e não encontraram diferença significativa entre a capacidade para o trabalho e capacidade funcional entre trabalhadores jovens $\mathrm{e}$ trabalhadores mais velhos. Em uma recente pesquisa, Alcântara et $\mathrm{al}^{11}$ encontraram uma relação direta positiva entre idade e capacidade para o trabalho, demostrando que os trabalhadores mais velhor relataram melhor capacidade para o trabalho. Segundo estes autores, esta relação positiva entre o envelhecimento e a capacidade para o trabalho pode ser atribuída à experiência adquirida pelos trabalhadores mais velhos. ${ }^{11}$

A manutenção da capacidade para o trabalho durante o processo de envelhecimento são, atualmente, um dos maiores desafios para a investigação no campo da saúde do trabalhador e estudos longitudinais que acompanham a evolução da capacidade para o trabalho durante todo o processo de envelhecimento podem esclarecer esta lacuna. No entanto, esse fato não exclui a importância e a necessidade de se adotar medidas de promoção da capacidade para o trabalho desses trabalhadores, uma vez que um trabalhador está inteiramente apto para desenvolver seu trabalho quando o escore de seu ICT for classificado como "ótimo".5,9 No caso dos resultados deste estudo em que a capacidade para o trabalho foi avaliada como boa é importante a adoção de medidas preventivas e de promoção da saúde, principalmente de ações que visem melhorar as condições, o ambiente e a organização do trabalho, bem como a saúde e a competência do trabalhador para atingir a capacidade para o trabalho considerada ótima.

A saúde e a capacidade funcional são importantes determinantes da capacidade para o trabalho do indivíduo. Os achados deste estudo confirmam a relação entre capacidade funcional e capacidade para o trabalho apontando que trabalhadores com melhor funcionalidade apresentam melhor capacidade para o trabalho. Estes resultados também apontam que a relação entre estas duas variáveis é dependente da idade, isto é, a correlação entre capacidade funcional e capacidade para o trabalho é mais forte em trabalhadores mais velhos. Dito de outra forma, a saúde e funcionalidade do trabalhador são fatores importantes para determinar a capacidade para o trabalho, principalmente em trabalhadores em fase de envelhecimento. Estes resultados corroboram evidências prévias sobre o papel da saúde na determinação da capacidade para o trabalho. Nygard et $\mathrm{al}^{10}$ observaram correlação significativa entre capacidade funcional com capacidade para o trabalho de trabalhadores idosos municipais. Lillefjell ${ }^{18} \mathrm{em}$ seu estudo sobre capacidade funcional e capacidade para o trabalho, observou forte correlação entre essas duas variáveis, e concluiu que a capacidade funcional é muito importante na prevenção de incapacidades para o trabalho. Padula et $\mathrm{al}^{17}$ também observou correlação entre essas variáveis em trabalhadores com mais de 50 anos. Alcântara et $\mathrm{al}^{11}$ encontraram uma relação positiva entre saúde e capacidade para o trabalho, sugerindo que trabalhadores com melhor avaliação funcional apresentam maior capacidade para o trabalho. Da mesma forma, o presente estudo observou que essas variáveis têm correlação moderada entre os trabalhadores mais jovens e forte entre trabalhadores com mais de 45 anos, confirmando a relação significativa entre funcionalidade e capacidade para o trabalho já preconizada pela OMS.

\section{CONSIDERAÇÕES FINAIS}

Algumas limitações do presente estudo devem ser ressaltadas: a impossibilidade de se estabelecer causalidade em estudos transversais e a importância do desenvolvimento de estudos longitudinais para acompanhar as mudanças na capacidade para o trabalho e na capacidade funcional da população trabalhadora, principalmente entre trabalhadores em processo de envelhecimento; o uso de uma amostragem por conveniência que não permite generalizar os resultados desta pesquisa e pode apresentar viés em termos de caracterização dos trabalhadores. No entanto, apesar destas considerações, foi observado que o perfil da amostra se assemelha ao perfil dos trabalhadores brasileiros em termo de escolarização, idade e gênero.

Como resultados, este estudo observou capacidade para o trabalho considerada boa e os trabalhadores apresentaram grau leve ou nenhuma incapacidade funcional. Também não houve diferença significativa entre capacidade funcional e capacidade para o trabalho nos trabalhadores, quando comparados em relação à idade, sugerindo que trabalhadores mais velhos mostram-se ativos e sem restrições para desenvolverem suas atividades laborais. Por fim, este estudo indicou uma correlação significativa entre capacidade para o trabalho e capacidade funcional, reforçando a relação entre saúde e desempenho nas atividades laborais.

Estes resultados apontam para a valorização do trabalhador idoso que mantém bom nível de funcionalidade e capacidade de desenvolver suas atividades no trabalho tão bem quanto os trabalhadores jovens. 0 Brasil tem apresentado um dos processos mais agudos de envelhecimento populacional do mundo e a permanência da pessoa que envelhece no mercado de trabalho se torna cada vez mais emergente. Pensando nesse fenômeno, é importante otimizar a capacidade para o trabalho e a capacidade funcional dos trabalhadores jovens e dos trabalhadores em fase de envelhecimento que continuamente irão compor parte da população economicamente ativa no Brasil, trazendo para o país um grande acúmulo de capital, associado a um envelhecimento saudável ao longo da vida.

\section{Agradecimentos}

RBC Valério trabalhou na concepção teórica, elaboração, coleta e análise dos dados e discussão dos resultados; FCM Silva e Dutra trabalhou na orientação, elaboração, análise dos dados e redação final do texto. 


\section{REFERENCIAS}

1. Instituto Brasileiro de Geografia e Estatística-IBGE. Sintese de Indicadores Sociais: Uma análise das condições da População Brasileira. Brasilia: IBGE; 2013.

2. Souza RF, Matias HA, Brêtas ACP. Reflexões sobre envelhecimento e trabalho. Ciên Saúde Colet. 2010; 15(6):2835-2843.

3. Costa G, Sartori S. Ageing, work hours and work ability. Ergonomics. 2007; 50(11):1914-1930.

4. Banco Mundial. Envelhecendo em um Brasil mais velho: implicações do enveIhecimento populacional para o crescimento econômico, a redução da pobreza, as finanças públicas e a prestação de serviços. Brasilia, DF: The World Bank; 2011.

5. Tuomi K, IImarinen J, Jahkola A, Katajarinne L, Tulkki A. Índice de capacidade para o trabalho. São Paulo: EdUFSCar; 2005.

6. Sjögren-Rönkä T, Ojanen MT, Leskinen EK, Tmustalampi S, Mälkiä EA. Physical and psychosocial prerequisites of functioning in relation to work ability and general subjective well-being among office workers. Scand J Work Env Hea. 2002; 28(3):184-90.

7. Silva LG, Haddad MCL, Domansky RC, Vitun DW. Capacidade para o trabalho entre trabalhadores de higiene e limpeza de um hospital universitário público. Rev Eletr Enf (Goiânia). 2010; 12(1):58-63.

8. Martinez MC, Latorre MRDO, Fischer FM. Capacidade para o trabalho: revisão de literatura. Ciên Saúde Colet. 2010; 15(supl.1):1553-1561.

9. Sampaio RF, Augusto VG. Envelhecimento e trabalho: um desafio para agenda da reabilitação. Rev Bras Fisioter. 2012; 16(2):94-101.

10. Nygard CH, Eskelinen L, Suvanto S, Tuomi K, IImarinen J. Associations between functional capacity and work ability among elderly municipal employees. Scand J Work Environ Health. 1991;17(supl.1):122-127.
11. Alcântara MA, Sampaio RF, Assunção AA, Silva FCM. Work Ability: Using structural equation modeling to assess the effects of aging, health and work on the population of Brazilian municipal employees. Work. 2014; 49(3):465-72.

12. Silveira C, Parpinelli MA, Pacagnella RC, Camargo RS, Costa ML, Zanardi DM, et al. Cross-cultural adaptation of the World Health Organization Disability Assessment Schedule (WHODAS 2.0) into Portuguese. Rev Assoc Med Bras. 2013; 59(3):234-240.

13. Gomes R, Nascimento EF, Araujo FC. Por que os homens buscam menos os serviços de saúde do que as mulheres? As explicações de homens com baixa escolaridade e homens com ensino superior. Cad Saúde Pública. 2007; 23(3):565-574.

14. nstituto Brasileiro de Geografia e Estatística-IBGE. Pesquisa mensal de emprego - PME. Mulher no mercado de trabalho: perguntas e respostas. Brasilia: IBGE; 2012.

15. Nonato FJAP, Pereira RHM, Nascimento PAMM, Araújo TC. 0 perfil da força de trabalho brasileira: Trajetórias e perspectivas. Brasilia: IPEA; 2012. (Mercado de trabalho; 51).

16. Moura AL, Reis LM, Vannuchi MTO, Haddad MCL, Domansky RC. Capacidade para o trabalho de funcionários da prefeitura de um campus universitário público. Rev Eletr Enf (Goiânia). 2013;15(1):130-137.

17. Padula RS, Comper ML, Moraes SA, Sabbagh C, Pagliato WJ, Perracini MR. The work ability index and functional capacity among older workers. Braz J Phys Ther. 2013; 17(4):382-391.

18. Lillefjell M. Function and Work Ability Following Multidisciplinary rehabilitation for Individuals with Chronic Musculoskeletal Pain [dissertation]. Trondheim: Norwegian University of Science and Technology-Department of Social Work and Health Science; 2007. 